Review Article

\title{
Update on PARP inhibitor therapy for solid tumors
}

\begin{abstract}
Poly (ADP-ribose) polymerases (PARPs) play an important role in DNA damage repair. They are primarily involved in base excision repair in single strand breaks. So far, the clinical trial results are very promising in breast and ovarian cancer with deleterious germline BRCA1 and BRCA2 mutations, and their use is expanding to include other solid tumors with homologous recombination (HR) repair defect. Studies suggest a correlation between tumor sensitivity to platinums and response to PARP inhibitors (PARPi) in women with ovarian cancer. The hypothesis is that by interfering with DNA repair, PARPi sensitize cells to DNA-damaging chemotherapies and radiation therapy. This article provides an overview of clinical trial results obtained with PARPi in the treatment of breast, ovarian, prostate, gastric, pancreatic, and lung cancers. In addition, we review resistance mechanisms to PARPi, toxicities of PARPi, and potential treatment combinations with PARPi.
\end{abstract}

Keywords: BRCA1, BRCA2, breast cancer, ovarian cancer, niraparib, olaparib, PARP inhibitor, rucaparib, talazoparib, veliparib
Volume 10 Issue 4 - 2019

\author{
Soley Bayraktar, ${ }^{1,2}$ Stefan Glück, ${ }^{3}$ HS Darling ${ }^{4}$ \\ 'Department of Medicine, Division of Medical Oncology and \\ Hematology, Countryside Hospital, USA \\ ${ }^{2}$ Department of Medicine, Division of Medical Oncology and \\ Hematology, Biruni University School of Medicine, Turkey \\ ${ }^{3}$ Vice President Global Medical Affairs, Celgene Corporation, \\ USA \\ ${ }^{4}$ Department of Medical Oncology and Hemato-oncology, \\ Narayana Superspeciality Hospital, India
}

Correspondence: Soley Bayraktar, Mayo Clinic Health system, Albert J and Judith A. Dunlap Cancer Center, I22I Whipple St, Eau Claire,WI, 54702, USA, Fax 7I5-464-8I0I, Tel 903-78I1580, Email soley.bayraktar@gmail.com

Received: September 25, 2019 | Published: October 03, 2019
Abbreviations: SSB, single-strand break; HR, homologous recombination; NHEJ, non-homologous end joining; PARPi, PARP inhibitors; PFS, progression-free survival; CT, care chemotherapy; CI, confidence interval; TNBC, triple-negative breast cancer; FDA, food and drug administration; G-CSF, granulocyte colony stimulating factors

\section{Introduction}

\section{PARP function in DNA damage repair}

There are different types of DNA defects caused by chemicals and environmental factors which include single-strand break
(SSB), mismatch repair (MMR), and double-strand break (DSB). ${ }^{1}$ Polyadenosine diphosphate [ADP] ribose polymerase-1 (PARP1) and -2 (PARP2) enzymes ${ }^{2}$ are responsible for repair of most of the SSB. After they detect the defective site, they bind to the DNA damage site and recruit a set proteins to repair the break. 3,4 When those proteins are recruited to the damaged site, the PARP-DNA interaction becomes unstable so that DNA repair can proceed. ${ }^{5}$ If the SSB are not repaired, they are converted to DSB. ${ }^{6}$ In that situation, another repair mechanism called homologous recombination (HR) will play a role. This mechanism is well represented in PARP1 knockout mice that SSB could not be repaired, but HR repair and non-homologous end joining (NHEJ) pathways were able to repair the formed DSB (Figure 1). HR is slower than NHEJ, but it is more accurate. ${ }^{7}$

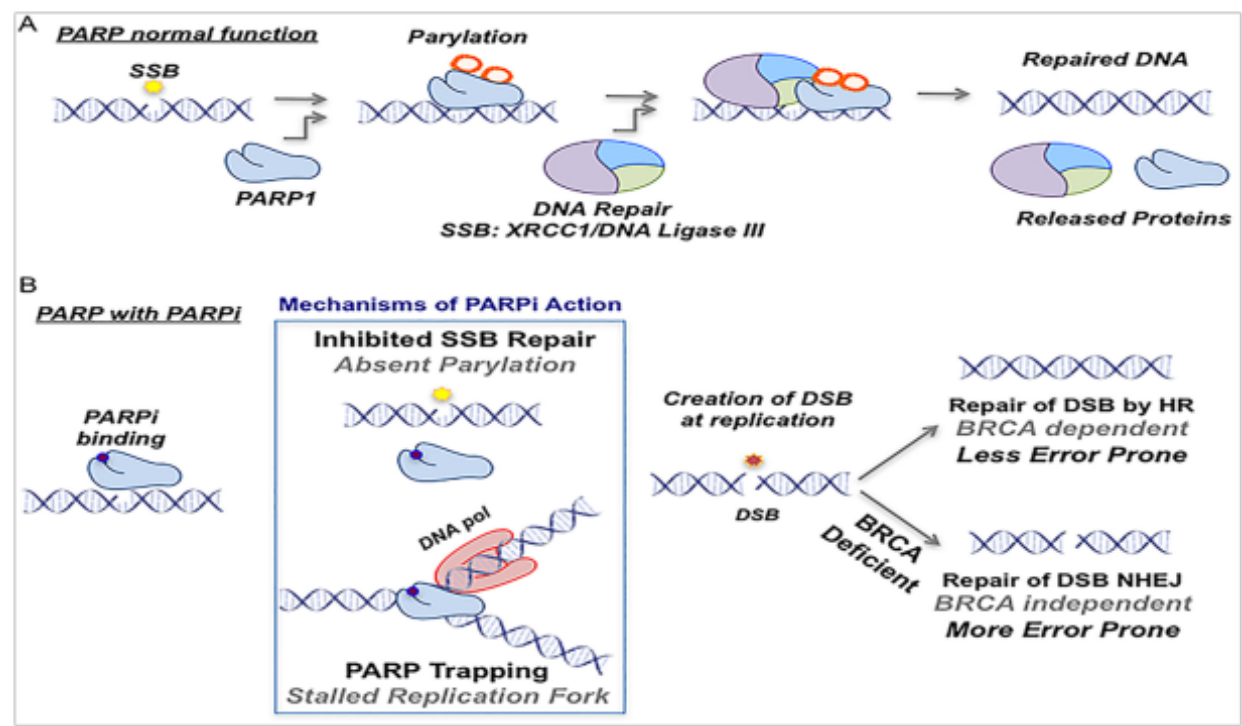

Figure I PARP function and mechanism (Adapted by Rimar et al, Cancer, 2017). 


\section{In homologous recombination and synthetic lethality}

As explained above, BRCA1 and BRCA2 proteins play an important role in the HR repair pathway. ${ }^{8}$ If they are dysfunctional, DSB can't be repaired. If both PARP and BRCA repair enzymes are insufficient, then DNA damage may become irreversible and result in apoptosis. ${ }^{9}$ Therefore, inhibition of PARP1 leads to very selective toxicity in BRCA1/2-deficient cells, but sparing the BRCA proficient cells, the so-called synthetic lethality (Figure 2 ).${ }^{10}$ The other example of synthetic lethality is seen with the platinum agents, where, interstrand cross linking caused by platinum agents is not adequately repaired by homologous recombination deficient cells leading to cell death through mitotic catastrophe or apoptosis. ${ }^{11}$

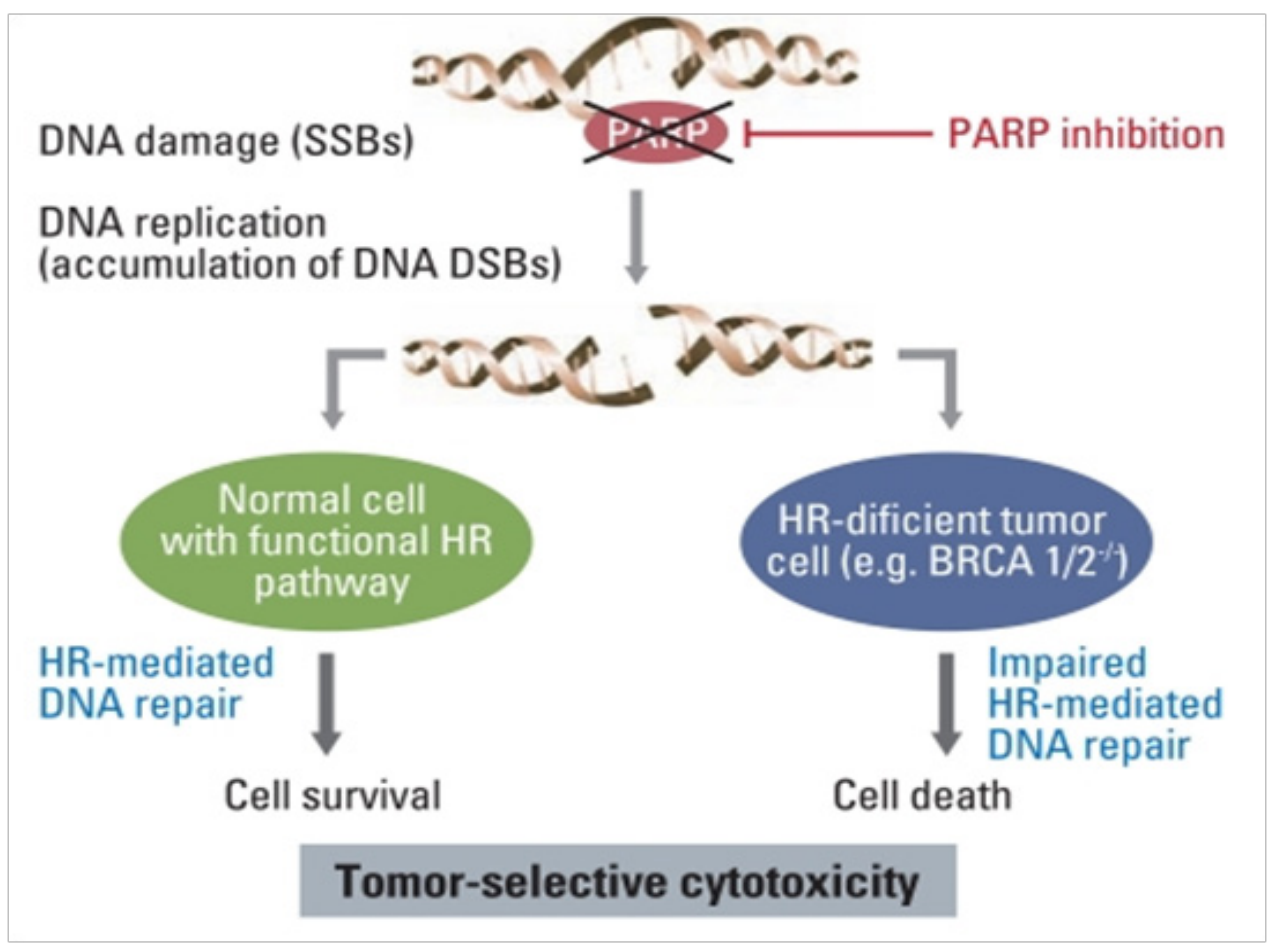

Figure 2 Mechanism for synthetic lethality in BRCAI/2 deficient cancer (Adapted by Saijo, Clin Ca Res, 2012).

\section{PARP inhibitors (PARPi) mechanism of action}

When PARPi are given to a patient, unrepaired DSBs will cause apoptosis and cell death. ${ }^{9}$ Another mechanism which has been attributed to PARPi were shown by Murai et al. ${ }^{12}$ in which PARPi creates an irreversible bond between PARP and DNA damaged site, known as "PARP-trapping" (Figure 1). This irreversible complex also will result in cell death. Recently, PARPi has also been shown to induce mitophagy (degradation of mitochondria by autophagy) and cell apoptosis. ${ }^{13}$

There are currently several PARPi in clinical development, including olaparib, veliparib, niraparib, rucaparib, and talazoparib all of which bind to both PARP1 and PARP2, but they have different potency in PARP trapping. ${ }^{14}$ Talazoparib is known as the most potent PARPi, thus requiring lower concentrations of talazoparib is enough to fully inhibit PARP-1 and $-2 \cdot{ }^{15}$ Rucaparib is another PARPi which is shown to inhibit tankyrase 1 and 2 as well as PARP $-4,-12,-15$, and $-16 .{ }^{16}$

\section{PARPi monotherapy in metastatic breast cancer (MBC)}

Olaparib: The first clinical trial conducted to assess the efficacy, safety, and tolerability of olaparib monotherapy in BRCA1 or BRCA2 mutation positive women with $\mathrm{MBC}$ who had received three or more lines of chemotherapy previously. Overall response rates (ORR) ranged from $22 \%$ to $41 \%$ with favorable toxicity profile. ${ }^{17}$ However, a phase II study evaluating olaparib monotherapy $400 \mathrm{mg}$ twice a day among patients with $\mathrm{MBC}(\mathrm{n}=26,81 \% \mathrm{TNBC})$ did not show any responses in neither germline BRCA1/2 (gBRCA1/2) mutation positive $(n=10)$ nor negative $(n=16)$ cases, even though the target lesions were reduced in size by $>30 \%$ in $50 \%$ patients with positive gBRCA1/2 mutations..$^{18}$ Following these discouraging results, finally came positive results from the phase III OlympiAD trial. In this trial, patients with gBRCA-mutated HER2-negative MBC who had received $\leq 2$ prior therapies were enrolled and olaparib monotherapy (300-mg PO BID) was compared to standard of care chemotherapy (CT) (vinorelbine, capecitabine or eribulin). ${ }^{19}$ After a median followup of 14.5 months, progression-free survival (PFS) was significantly longer with olaparib versus CT (7.0 vs 4.2 months; hazard ratio [HR], $0.58 ; 95 \%$ confidence interval $(\mathrm{CI}), 0.43-0.8 ; \mathrm{P}<0.001)$; ORR in the olaparib group was also higher $(59.9 \%$ vs $28.8 \%)$. On the other hand, the overall survival (OS) outcomes was not different $(19.3$ months in olaparib arm vs 19.6 months in CT arm; HR 0.90, 95\% CI: $0.63-1.29, \mathrm{p}=0.57)$. The greatest benefit from olaparib were seen in group of patients who were platinum-naïve and also in patients with triple-negative breast cancer (TNBC). In this trial, patients who had olaparib had fewer grade $3 / 4$ toxicities compared to CT $(16.1 \%$ vs $4.4 \%)$. After these encouraging results, Based on these results, Food and Drug Administration (FDA) approved olaparib in January 2018 
for gBRCA1/2-mutated HER2-negative MBC setting after 1 prior line of chemotherapy, or endocrine therapy if they have ER-positive MBC.

Talazoparib: The EMBRACA study is a recently reported phase III clinical trial comparing talazoparib, $1 \mathrm{mg}$ orally daily to CT (eribulin, vinorelbine, capecitabine, or gemcitabine) in patients with gBRCAmutated MBC who had $\leq 3$ lines of chemotherapy in the advanced setting. ${ }^{20}$ Median PFS was significantly prolonged with talazoparib versus $\mathrm{CT}$ (8.6 vs 5.6 months; $\mathrm{HR}, 0.54 ; \mathrm{P}<0.0001$ ), and the ORR was superior $(62 \%$ vs $27 \%$; HR, $5.0 ; \mathrm{P}<0.0001)$. The HR for death was $0.76(95 \% \mathrm{CI}, 0.55$ to $1.06 ; \mathrm{P}=0.11)$. Any hematologic grade $3 / 4$ adverse events were more common in PARPi group vs chemotherapy group (55\% vs $38 \%$ ), including grade 3 or 4 anemia (39.2\% vs $4.8 \%$ ) and thrombocytopenia ( $15 \%$ vs $2 \%$ ); only neutropenia was less common with talazoparib ( $21 \%$ vs $35 \%$ ). In contrast to OlympiAD trial, all subsets of patients benefited from talazoparib, ER-positive vs TNBC, platinum-naïve vs not, presence or absence of brain metastases, or BRCA mutation status. In October 2018, The FDA approved talazoparib for $\geq 1$ st line monotherapy for gBRCA-mutated HER2-negative MBC.

Niraparib and Rucaparib: Niraparib has been tested in two phase III clinical trials, one of which is looking at single- agent niraparib therapy versus $\mathrm{CT}$ for gBRCA-mutated HER2-negative $\mathrm{MBC}$ patients who had $\geq 2$ lines of CT in metastatic setting (BRAVO trial) (Table 1). At the present time, rucaparib is in early phases of development. Rucaparib is undergoing single arm phase II trial NCT02505048 (RUBY) as a monotherapy in HER2-negative MBC patients who had $\geq 1$ lines of CT in the metastatic setting, and who carry a BRCAness tumor profile which was defined as "high tumor genomic LOH" score and/or a somatic BRCA mutation, but no BRCA1/2 germline mutations.

Table I selected phase III trials in breast cancer

\begin{tabular}{|c|c|c|c|c|}
\hline \multicolumn{5}{|c|}{ Advanced/metastatic setting } \\
\hline & Study & Design & Population & Results \\
\hline \multirow[t]{4}{*}{ Olaparib } & OlympiAD & Olaparib vs PCT & gBRCA,$\leq 2$ prior lines & PFS: 7 vs $4.2 \operatorname{mos}(P<0.00 \mathrm{I})$ \\
\hline & & & & ORR: $59.9 \%$ vs $28.8 \%(P<0.00 I)$ \\
\hline & & & & OS: 19.3 vs $19.6 \operatorname{mos}(p=N S)$ \\
\hline & LUCY & Single arm olaparib & $\begin{array}{l}\text { gBRCA }, \leq \mathrm{I} \text { prior lines containing } \\
\text { taxane or anthracycline }\end{array}$ & Active and recruiting \\
\hline Veliparib & BROCADE 3 & $\begin{array}{l}\mathrm{C}+\mathrm{P}+\mathrm{V} \rightarrow \text { maintenance } \mathrm{V} \text { vs } \\
\mathrm{C}+\mathrm{P}+\text { placebo }\end{array}$ & gBRCA, $\leq 2$ lines of therapy & Active, not recruiting \\
\hline \multirow[t]{3}{*}{ Talazoparib } & EMBRACA & Talazoparib vs PCT & gBRCA,$\leq 3$ prior lines & PFS: 8.6 vs $5.6 \operatorname{mos}(P<0.000 \mathrm{I})$ \\
\hline & & & & ORR: $62 \%$ vs $27 \%(\mathrm{P}<0.000 \mathrm{I})$ \\
\hline & & & & $\begin{array}{l}\text { Interim median } H R \text { for death: } \\
0.76(P=0.1 I)\end{array}$ \\
\hline Niraparib & BRAVO & Niraparib vs PCT & gBRCA,$\leq 2$ prior lines & Active, not recruiting \\
\hline \multicolumn{5}{|c|}{ Neoadjuvant setting } \\
\hline \multirow[t]{5}{*}{ Veliparib } & BrighTNess & $\mathrm{C}+\mathrm{P}+\mathrm{V}(\mathrm{CPV}) \rightarrow \mathrm{AC}$ & TNBC & $\begin{array}{l}\text { PCR: CPV: } 53 \% \text { vs CPP: } 57 \% \\
(p=N S)\end{array}$ \\
\hline & & vs & & \\
\hline & & $\mathrm{C}+\mathrm{P}+$ placebo $(\mathrm{CPP}) \rightarrow \mathrm{AC}$ & & \\
\hline & & vs & & \\
\hline & & Placebo+P $\rightarrow A C$ & & \\
\hline \multirow[t]{2}{*}{ Olaparib } & PARTNER & Olaparib+weekly P+C & TNBC or gBRCA, & Active and recruiting \\
\hline & SUBITO & $\begin{array}{l}\mathrm{ddAC} \rightarrow \mathrm{C}+\mathrm{P} \longrightarrow \text { olaparib } \\
\text { maintenance }\end{array}$ & $\begin{array}{l}\text { stage III, HER2(-), BRCAI-like } \\
\text { breast cancer patients }\end{array}$ & Active and recruiting \\
\hline \multicolumn{5}{|c|}{ Adjuvant setting } \\
\hline Olaparib & OlympiA & Olaparib vs Placebo & $\begin{array}{l}\text { gBRCA, post-completion SOC } \\
\text { neo/adjuvant therapy }\end{array}$ & Active, not recruiting \\
\hline
\end{tabular}

Abbreviations: AC, doxorubicin+cyclophosphamide; C, carboplatin; V, veliparib; gBRCA, germline BRCA mutated; P, paclitaxel; PCT, physician's choice of chemotherapy; SOC, standard of care;TNBC, triple-negative breast cancer; CTC, cyclophosphamide $3000 \mathrm{mg} / \mathrm{m}^{2}$ day I mesna $500 \mathrm{mg}$ (push) $+2000 \mathrm{mg}$ in 24 hours day I carboplatin $\left(400 \mathrm{mg} / \mathrm{m}^{2}\right.$; days I,2 thiotepa $250 \mathrm{mg} / \mathrm{m}^{2}$ day 2 ; NS, non-significant 


\section{PARPi monotherapy in early stage breast cancer}

Olaparib: OlympiA (NCT02032823) is a phase III trial evaluating olaparib at $300 \mathrm{mg}$ PO BID for 1 year in patients with high-risk stage 2/3 gBRCA1/2 mutated breast cancer. The OlympiA trial has now completed enrollment and results are awaited.

Talazoparib: I neoadjuvant setting, talazoparib was given for 2 months to 13 patients with early-stage gBRCA1/2 associated breast cancer. Overall response rate was almost $100 \% .{ }^{21}$ This study is now evaluating extended neoadjuvant talazoparib therapy.

\section{Combining PARPi with chemotherapy in breast cancer}

Myelosuppression is the primary dose-limiting toxicity for PARPi, which has made combination of PARPi with cytotoxic chemotherapies difficult. Veliparib has been considered the most suitable PARPi to combine with cytotoxic agents because of its modest hematotoxicity. Different strategies have been employed to mitigate the myelosuppressive effects of PARPi: PARPi dose reduction, intermittent dosing schedules, and support with granulocyte colony stimulating factors (G-CSF). It is not currently clear which one is a more viable therapeutic option, but the results of the phase III BrighTNess trial (discussed later) suggests that using subtherapeutic doses of PARPi in combination with standard dosages of cytotoxic chemotherapeutic agents does not significantly improve clinical outcomes.

Veliparib plus chemotherapy: The results of the 3-arm, randomized, placebo-controlled phase III BrighTNess trial which evaluated carboplatin/taxol plus veliparib versus carboplatin/taxol versus taxol alone followed by 4 cycles of doxorubicin and cytoxan chemotherapy given neoadjuvantly to patients with TNBC. This trial did not show any benefit from addition of veliparib (pCR rates were $53 \%$ vs $57 \%$, respectively). ${ }^{22}$ It should be noted that paclitaxel, $80 \mathrm{mg} / \mathrm{m}^{2}$, and carboplatin AUC6 were standard full doses, but the veliparib dose is $1 / 8$ of the monotherapy dose of veliparib, $400 \mathrm{mg}$ twice daily.

In phase II BROCADE study, Han et al. ${ }^{23}$ evaluated the safety and efficacy of addition of veliparib to either temozolomide (VT) or carboplatin/paclitaxel (VCP) versus carboplatin/paclitaxel alone (PCP) in gBRCA-mutated MBC patients. In contrast to BrighTNess trial, ORR was much better after the addition of veliparib $(77.8 \%$ vs $61.3 \% ; \mathrm{P}=.027$ ), but survival outcomes were not different. An ongoing phase III BROCADE trial is testing VCP vs PCP, followed by maintenance veliparib in this patient population. The veliparib dose is $120 \mathrm{mg}$ PO BID and is only given on days $1-7$ of every 4 week cycle. We eagerly await results from this trial.

Olaparib plus chemotherapy: The placebo-controlled PARTNER trial (NCT03150576) is a phase III trial testing the combination of olaparib and weekly taxol and carboplatin AUC 5 in neoadjuvant setting among patients with TNBC and/or gBRCA mutated breast cancer. Stage 1 accrual is complete and stage 2 began in August 2017.

The phase II NCT01074970 trial is evaluating 2-year DFS among patients with gBRCA-mutated TNBC treated with single agent cisplatin versus cisplatin plus rucaparib following neoadjuvant systemic therapy. SUBITO is a randomized, (neo)adjuvant phase III trial in stage III, HER2-negative, germline BRCA1 or BRCA 2 mutated or BRCA1-like breast cancer patients, comparing standard of care chemotherapy followed by one year of adjuvant olaparib and high dose chemotherapy. Table 1 shows the most important randomized phase III trials investigating the use of PARPi, either as a single agent versus combined with chemotherapy in different settings.

\section{Combining PARPi with immunotherapy in breast cancer}

Combining immunotherapy with PARPi is a promising approach because BRCA mutated cancers are more genomically unstable due to the defect in HR, and therefore may be more immunogenic. The expected cross-toxicity is also limited.

Olaparib plus immunotherapy: Olaparib plus durvalumab in patients with advanced solid tumors ${ }^{24}$ who had prior therapy with anthracycline/taxane/platinum-containing chemotherapy regimens was tested in MEDIOLA trial is a phase I/II trial. Patients received olaparib, 300mg orally twice daily for 4 weeks which was followed by olaparib plus durvalumab combined therapy. Of 25 patients enrolled, the ORR was $67 \%$ in patients who had this regimen as a first line therapy. Median PFS has not been reached at the time of presentation. Phase II DORA (NCT03167619) is studying olaparib in combination with durvalumab in platinum-treated metastatic TNBC.

Other PARPi plus immunotherapy: Additional immunotherapy and PARPi combination studies are also in development. Niraparib plus pembrolizumab is being studied in a phase I/II study among TNBC and ovarian cancer patients (TOPACIO/Keynote-162/NCT02657889). Phase II NCT02849496 will evaluate veliparib plus atezolizumab in gBRCA1/2+ TNBC patients. Phase Ib/II JAVELIN PARP MEDLEY (NCT03330405) will enroll patients with gBRCA1/2+ or ATMmutation associated breast cancer for evaluation of talazoparib plus avelumab.

\section{PARP inhibition in ovarian cancer}

Approximately $41-50 \%$ of ovarian carcinomas are estimated to exhibit homologous recombination defect (HRD). ${ }^{25}$ However, the frequency of HRD varies among ovarian cancers associated with germline mutations versus somatic mutations, and according to the histological subtype. Pennington et al. ${ }^{26}$ found that defective HR genes (germline or somatic) are found in $31 \%$ of ovarian carcinomas. Elvin et al. ${ }^{27}$ evaluated the presence of BRCA mutations or loss of heterozygosity ( $\mathrm{LOH})$ in different histological subtypes. The serous subtype was associated with a higher prevalence of HRD, with $43.8 \%$ of the patients presenting BRCA mutations (gBRCAmut, 18.7\%) or BRCA wild-type/LOH-high (BRCAwt/LOH-high, 25.1\%). In relation to high-grade versus low-grade serous carcinoma, Norquist et al. ${ }^{28}$ found a vital difference in germline and somatic mutation rates of HR genes, which were $10.9 \%$ for low-grade versus $27 \%$ for highgrade serous ovarian carcinoma (HGSOC) (HR, 0.33; 95\% CI, 0.1$0.8 ; \mathrm{p}=0.02$ ).

Olaparib: Kaufman et al. ${ }^{29}$ evaluated efficacy of olaparib in 137 gBRCA-mutated recurrent ovarian cancer patients who had received $\geq 3$ lines of chemotherapy. The results were impressive in this heavily treated population. The reported ORR, PFS and OS were $34 \%, 7.9$ months and 16.6months, respectively. Most toxicities were manageable. On the basis of these data, FDA approved olaparib as a single agent in patients with advanced gBRCA-mutated ovarian cancer who had $\geq 3$ or more lines of chemotherapy.

In the SOLO 1 phase 3 trial, patients with newly diagnosed or platinum-sensitive stage III or IV high-grade serous or endometrioid ovarian cancer (HGSOC and HGEOC), primary peritoneal cancer, or fallopian-tube cancer with a germline or somatic BRCA1/2 mutations were given olaparib maintenance therapy. After a median follow-up of 41 months, HR for risk of death was 0.30 in olaparib arm compared 
to no maintenance therapy. ${ }^{30}$ In December 2018, olaparib maintenance was approved by FDA in this patient population. In order to identify the eligible patients for maintenance olaparib after first line platinumcontaining chemotherapy, BRCA somatic mutation testing may be done on the tumor biopsy sample, and if the BRCA somatic mutation is negative then, the presence of germline BRCA mutation must be identified by testing the peripheral blood.

In the phase III SOLO2 trial, patients with recurrent platinumsensitive gBRCA- mutated HGSOC and HGEOC also benefited from olaparib maintenance therapy (300 $\mathrm{mg}$ twice daily). The HR for PFS was 0.30 with an absolute gain in PFS of 13.6 months. ${ }^{31}$ Study 19 (NCT00753545) is a phase II randomized trial which enrolled patients with recurrent platinum sensitive HGSOC and HGEOC regardless of their BRCA status (1:1) to receive olaparib $400 \mathrm{mg}$ twice daily or placebo. In this study ${ }^{32}$ all benefits benefited from olaparib and HR for death was similarly reduced by $65 \%$ (HR 0.35 [95\% CI, $0.25-$ 0.49 ], $\mathrm{P}<0.0001$; with an absolute gain in PFS of 3.6 months. The absolute gain in OS was only 2 months, but it was statistically significant. In view of these results, in August 2017, FDA-approved olaparib maintenance for patients with recurrent platinum-sensitive epithelial ovarian cancer, fallopian tube, or primary peritoneal cancer, irrespective of their BRCA status. Although the PARP inhibitor (olaparib) maintenance therapy achieved substantial PFS benefit among patients with platinum-sensitive recurrent ovarian cancer after a CR/PR to the most recent regimen, the significantly improved PFS benefit did not translate into a major OS benefit in olaparib trials. The most likely explanation for the lack of OS benefit is that effective post-progression therapy overcomes this benefit.

The phase III SOLO- $3^{33}$ trial is evaluating the efficacy and safety of olaparib in patients with a deleterious BRCA1/2 mutations following two or more prior lines of chemotherapy. The 266 eligible patients were randomized (2:1) to receive olaparib $300 \mathrm{mg}$ twice daily or PCT (paclitaxel, topotecan, pegylated liposomal doxorubicin or gemcitabine). The results were recently presented at the 55th Annual Meeting of the American Society of Clinical Oncology (ASCO) in Chicago, on June 3rd 2019. The ORR in the olaparib arm was superior compared to the chemotherapy arm (ORR; $72.2 \%$ for olaparib vs $51.4 \%$ for chemotherapy; $95 \%$ CI: 1.40 to $4.58 ; \mathrm{p}=0.002$ ). The PFS was also significantly increased in the olaparib arm (13.4 months) compared to the chemotherapy arm (9.2 months; PFS HR, 0.62, $\mathrm{p}=0.013$ ).

Rucaparib: The phase II ARIEL trial ${ }^{34}$ enrolled patients with platinum sensitive advanced ovarian cancer who were previously treated with two or more lines of chemotherapy. HRD was assessed by evaluating both BRCA germline mutations and $\mathrm{LOH}$. Patients were divided into: BRCA mutant (deleterious germline or somatic), BRCA wild type with loss of heterozygosity ( $\mathrm{LOH}$ ) high, or BRCA wild-type with LOH low, using a cutoff of $14 \%$ or more genomic $\mathrm{LOH}$ for $\mathrm{LOH}$ high. The ORR and PFS were higher in gBRCA carriers (RR, 69\%; PFS, 12.8 months; HR, 0.27; 95\% CI, 0.16-0.44; $\mathrm{p}=0.0001$ ) and BRCA wild-type LOHhigh patients (RR, 39\%; PFS, 5.7months; HR, 0.62; 95\% CI, 0.42$0.9 ; \mathrm{p}=0.011)$ than in BRCA wild-type/LOH-low patients $(\mathrm{RR}, 11 \%$;
PFS, 5.1months). Based on these results, in 2016, the FDA approved rucaparib monotherapy for patients with platinum sensitive, relapsed or progressive, BRCA mutated (germline or somatic) HGSOC or HGEOC, fallopian tube, or primary peritoneal cancer, who have been treated with two or more prior lines of platinum-based chemotherapy, and who are unable to tolerate further platinum based chemotherapy.

The phase III ARIEL 3 trial evaluated the maintenance of rucaparib versus placebo following a response to second line or later platinum-based chemotherapy in different patient subgroups. ${ }^{35}$ PFS in 3 subgroups were significantly better: BRCA-mutant tumors compared to placebo treated patients (gBRCA mutation, 16.6months vs. 5.4 months); patients with HRD (including gBRCA mutation and BRCAwt/high-LOH carcinomas) (median PFS, 13.6 months vs 5.4 months; HR, $0.32 ; 95 \% \mathrm{CI}, 0.24-0.42 ; \mathrm{p}=0.0001)$; and the intention-to-treat population (median PFS, 10.8 months versus 5.4 months; HR, 0.36; 95\% CI, 0.30-0.45; $\mathrm{p}=0.0001$ ). Based on these data, on 6 April 2018, FDA expanded rucaparib indications to the maintenance treatment of recurrent epithelial ovarian, fallopian tube, or primary peritoneal cancers achieving a complete or partial response to platinum-based chemotherapy. Clinical trials specifically on PARP inhibitor maintenance therapy for non-BRCA1/2-mutated ovarian cancer should be initiated as soon as possible to augment the understanding of the benefits of PARP inhibitor maintenance therapy and inform the use of PARP inhibitors in this patient population.

Niraparib: FDA approved niraparib for maintenance therapy of recurrent platinum sensitive ovarian, fallopian tube, or primary peritoneal cancer, regardless of BRCA status based on the phase III ENGOT-OV16/NOVA trial. ${ }^{36}$ In the NOVA trial, niraparib significantly increased median PFS in platinum-sensitive ovarian cancer independent of HRD and gBRCA mutations. A total of 553 patients were divided into two main cohorts: gBRCA and non-germline BRCA mutated. In gBRCA mutant group, PFS was significantly longer with niraparib compared to placebo (21.0 months vs. 5.5 months; HR = $0.27,95 \%$ CI: $0.17-0.4)$. When HRD tumors were retrospectively analyzed in an exploratory analysis out of the non-gBRCA group, the PFS of patients with BRCA wild-type/HRD-high (20.9 months vs 11.0 months; hazard ratio, $0.27 ; 95 \%$ CI, 0.08-0.90) was increased to a greater extent than that of BRCA-wild-type/HRD-low patients (6.9months vs 3.8months; hazard ratio, $0.58 ; 95 \%$ CI, 0.36-0.92). Fabbro et al. ${ }^{37}$ recently resported the safety and efficacy of niraparib in older patients ( $\geq 70$ years of age) which were comparable to the results in younger population.

Selected phase II/III clinical trials of PARPs inhibitors for treatment of ovarian cancer are summarized in Table 2. In summary, olaparib demonstrated high efficacy as maintenance treatment in first line (SOLO1) and subsequent lines (SOLO2) BRCA1/2 mutationassociated and in subsequent lines of therapies (Study 19) for platinum sensitive ovarian cancer patients with or without BRCA mutation. Niraparib (ENGOTOV16/ NOVA) and rucaparib (ARIEL 3 ) are effective in maintenance setting for platinum sensitive recurrent ovarian cancer regardless of BRCA status.

Table 2 Selected phase II/III trials in ovarian cancer

\begin{tabular}{|c|c|c|c|c|}
\hline & Study & Design & Population & Results \\
\hline \multirow[t]{2}{*}{ Olaparib } & SOLO I & $\begin{array}{l}\text { Olaparib } 300 \mathrm{mg} \text { BID } \\
\text { maintenance vs placebo }\end{array}$ & $\begin{array}{l}\text { Platinum sensitive HGSOC after first line platinum based CT, } \\
\text { germline or somatic BRCAmut }\end{array}$ & Median PFS: NR vs I3.8 mo $(p<0.001)$ \\
\hline & SOLO 2 & $\begin{array}{l}\text { Olaparib } 300 \mathrm{mg} \text { BID } \\
\text { maintenance vs placebo }\end{array}$ & $\begin{array}{l}\text { Platinum sensitive recurrent HGSOC or HGEOC, primary } \\
\text { peritoneal or fallopian tube cancer, gBRCAmut }\end{array}$ & Median PFS: 19.1 vs $5.5 \mathrm{mo}(p<0.000 \mathrm{I})$ \\
\hline
\end{tabular}


Table continued

\begin{tabular}{|c|c|c|c|c|}
\hline & Study & Design & Population & Results \\
\hline & SOLO 3 & $\begin{array}{l}\text { Olaparib } 300 \mathrm{mg} \text { vs } \\
\text { PCT(NCT02282020) }\end{array}$ & $\begin{array}{l}\text { Platinum sensitive recurrent HGSOC or HGEOC, primary } \\
\text { peritoneal or fallopian tube cancer, germline or somatic } \\
\text { BRCAmut }\end{array}$ & $\begin{array}{l}\text { ORR: } 72.2 \% \text { for olaparib vs } 5 \mid .4 \% \text { PCT } \\
(p=0.002) \\
\text { PFS: I } 3.4 \text { vs } 9.2 \mathrm{mo}(p=0.013)\end{array}$ \\
\hline & Study 19 & $\begin{array}{l}\text { Olaparib } 400 \mathrm{mg} \text { BID } \\
\text { maintenance vs placebo }\end{array}$ & $\begin{array}{l}\text { Platinum sensitive recurrent HGSOC or HGEOC, primary } \\
\text { peritoneal or fallopian tube cancer, germline or somatic } \\
\text { BRCAmut }\end{array}$ & $\begin{array}{l}\text { PFS: } 8.4 \text { vs } 4.8 \mathrm{mo}(p<0.000 \mathrm{I}) \text { OS: } 29.8 \\
\text { vs } 27.8 \mathrm{mo}(\mathrm{HR}, 0.73)\end{array}$ \\
\hline & $\begin{array}{l}\text { PAOLA-I } \\
\text { (NCT02477644) }\end{array}$ & $\begin{array}{l}\text { Olaparib vs Placebo } \\
\text { plus Carboplatin/ } \\
\text { Paclitaxel/ Bevacizumab } \\
\text { as primary treatment and } \\
\text { maintenance }\end{array}$ & $\begin{array}{l}\text { Advanced FIGO stage IIIB - IV HGSOC or HGEOC, fallopian } \\
\text { tube, or peritoneal cancer }\end{array}$ & ongoing \\
\hline \multirow[t]{4}{*}{ Rucaparib } & ARIEL 2 PART I & Rucaparib 600mg BID & $\begin{array}{l}\text { Platinum sensitive recurrent HGSOC or HGEOC, primary } \\
\text { peritoneal or fallopian tube cancer }\end{array}$ & $\begin{array}{l}\text { Median PFS: BRCAmut: } 12.8 \mathrm{mo} \\
(p<0.000 \mathrm{I}) \\
\text { BRCAwt LOH high:5.7 mo }(p=0.01 \mathrm{I}) \\
\text { BRCAwt LOH low: } 5.2 \mathrm{mo}(p=0.01 \mathrm{I})\end{array}$ \\
\hline & ARIEL 3 & $\begin{array}{l}\text { Rucaparib } 600 \mathrm{mg} \text { BID } \\
\text { maintenance vs placebo }\end{array}$ & $\begin{array}{l}\text { Platinum sensitive recurrent HGSOC or HGEOC, primary } \\
\text { peritoneal or fallopian tube cancer }\end{array}$ & $\begin{array}{l}\text { Median PFS: BRCAmut: } 16.6 \text { vs } 5.4 \\
\text { mo }(p<0.000 \text { I }) \text { HRD+: } 13.6 \text { vs } 5.4 \\
\text { mo }(p<0.000 \text { I) ITTP: } 10.8 \text { vs } 5.4 \text { mo } \\
(p<, 0.0001)\end{array}$ \\
\hline & ARIEL 4 & $\begin{array}{l}\text { Rucaparib } 600 \mathrm{mg} \text { BID } \\
\text { vs PCT }\end{array}$ & $\begin{array}{l}\text { Platinum sensitive or resistant recurrent HGSOC or HGEOC, } \\
\text { primary peritoneal or fallopian tube cancer }\end{array}$ & ongoing \\
\hline & NCT01968213 & $\begin{array}{l}\text { Switch Maintenance } \\
\text { Monotherapy }\end{array}$ & HGSOC or HGEOC, primary peritoneal or fallopian tube cancer & ongoing \\
\hline Niraparib & NOVA & $\begin{array}{l}\text { Niraparib } 300 \mathrm{mg} \\
\text { maintenance vs placebo }\end{array}$ & $\begin{array}{l}\text { Platinum sensitive recurrent HGSOC; primary peritoneal or } \\
\text { fallopian-tube cancer }\end{array}$ & $\begin{array}{l}\text { Median PFS: gBRCAmut: } 2 \text { I vs } 5.5 \text { mo } \\
(p<0.00 \text { I }) \text { BRCAwt HRD+: } 12.9 \text { vs } 3.8 \\
\text { mo }(p<0.00 \text { I }) \\
\text { Overall non-gBRCA: } 9.3 \text { vs } 3.9 \text { mo } \\
(p<0.00 \text { I })\end{array}$ \\
\hline Veliparib & NCT02470585 & $\begin{array}{l}\text { Veliparib with Carboplatin } \\
\text { and Paclitaxel and as } \\
\text { continuation maintenance } \\
\text { therapy }\end{array}$ & $\begin{array}{l}\text { Newly diagnosed FIGO stage IIIB - IV HGSOC or HGEOC, } \\
\text { fallopian tube, or peritoneal cancer }\end{array}$ & ongoing \\
\hline
\end{tabular}

Abbreviations: PTS, patients; HGSOC, high grade serous ovarian cancer; OC, ovarian cancer; HGEOC, high-grade endometrioid cancer; BRCA mut, BRCA mutated; CT, chemotherapy; HRD+, homologous recombination deficiency positive; PFS, progression free survival; ORR, objective response rate; DCR, disease control rate; MDR, median duration of response; gBRCA, germline BRCA mutated; BRCA wt, BRCA wild type; LOH, loss of heterozygosity; ITTP, Intention to treat population; NR, not reached; $\mathrm{PCT}$, physician's choice of chemotherapy

Table 3 Selected phase III trials in pancreatic and prostate cancer

\begin{tabular}{|c|c|c|c|c|}
\hline & Study & Design & Population & Results \\
\hline \multirow[t]{2}{*}{ Olaparib } & POLO (NCT02 I84I95) & $\begin{array}{l}\text { Olaparib } 300 \text { mg BID vs } \\
\text { placebo }\end{array}$ & $\begin{array}{l}\text { gBRCA mutated pancreatic cancer whose } \\
\text { disease has not progressed on first line } \\
\text { platinum-based chemotherapy }\end{array}$ & $\begin{array}{l}\text { PFS: } 7.4 \text { vs } 3.8 \mathrm{mo}, \\
(p=0.004) \\
\text { OS: } 18.9 \text { vs } 18.1 \mathrm{mo} \text {, } \\
(p=0.68)\end{array}$ \\
\hline & PROFOUND & $\begin{array}{l}\text { Olaparib vs Enzalutamide vs } \\
\text { AA/prednisone }\end{array}$ & $\begin{array}{l}\text { mCRPC who failed prior AR-targeted } \\
\text { therapy }\end{array}$ & Active, not recruiting \\
\hline Rucaparib & TRITON & $\begin{array}{l}\text { Rucaparib vs Enzalutamide vs } \\
\mathrm{AA} / \text { prednisone vs docetaxel }\end{array}$ & $\begin{array}{l}\text { mCRPC who failed I prior AR-targeted } \\
\text { therapy }\end{array}$ & Active, not recruiting \\
\hline Talazoparib & TALAPRO & $\begin{array}{l}\text { Talazoparib+AR-targeted } \\
\text { therapy vs placebo+AR- } \\
\text { targeted therapy }\end{array}$ & $\mathrm{DRD}+\mathrm{mCRPC}$ & Active, recruiting \\
\hline
\end{tabular}

$\mathrm{AA}$, abiraterone; $\mathrm{mCRPC}$, metastatic castration resistant prostate cancer 


\section{PARP inhibition in prostate cancer}

Germline BRCA2 carriers have a 5 to 9 times more risk of developing prostate carcinoma. ${ }^{38}$ BRCA-associated prostate cancers are more aggressive than non-BRCA associated prostate cancers. ${ }^{39}$ Among all prostate cancers, $8-14 \%$ of them are associated with BRCA deleterious mutations. ${ }^{40,41}$ Additionally, $20 \%$ of all prostate cancers have deficient PTEN expression which can result in HR repair defects and make the tumor more susceptible to PARPi. ${ }^{42}$

PARPi olaparib was tested in 19 patients with advanced prostate cancer in the TOPARP-A trial (Trial of Olaparib in Patients with Advanced Castrate Resistant Prostate Cancer). ${ }^{43}$ This trial shed light into the subgroup of patients who may benefit from PARPi. According to this trial, $88 \%$ of patients who had a germline or somatic deletions mutations in BRCA1/BRCA2, ATM serine/threonine kinase (ATM), Checkpoint kinase 2 (CHEK2), or Fanconi anemia gene had a positive response to olaparib compared to only $6 \%$ of patients who did not have any alteration in any of those genes. ${ }^{44}$ After these results are published, FDA approved the use of olaparib in metastatic castration-resistant prostate cancer (CRPC) patients with BRCA1/2 or ATM alterations who have progressed on chemotherapy and at least one hormonal agent (abiraterone or enzalutamide). The phase 3 PROFOUND study was designed after these encouraging results are reported. This trial will include patients who had failed at least one hormonal agent and patients will be screened for somatic HR deficiency mutations and will then be randomized to either another AR-targeted therapy or olaparib.

The phase II trial TRITON2 (NCT02952534) is testing rucaparib $600 \mathrm{mg}$ twice daily in CRPC patients with a germline or somatic alteration in BRCA1/2 or HR deficiency who progressed on $\geq 1$ lines of AR-targeted therapy and 1 line of chemotherapy. ${ }^{45}$ According to the preliminary results presented at ESMO, $48 \%$ and $45 \%$ of BRCA carriers had a PSA response and radiological response, respectively. Now the continuation trial, the phase III TRITON3, will be evaluating rucaparib efficacy compared to physician's choice of abiraterone, enzalutamide, or docetaxel.

Molecular studies suggest that PARP1 may also be involved in regulation of $\mathrm{AR}$ activity at later stages of androgen-independent action. PARP1 activity is significantly increased in CRPC cells compared to hormone sensitive prostate cancer cells, and PARPi causes depletion of both the AR and PARP1 on chromatin in CRPC cells. ${ }^{46}$ Mathew et al. ${ }^{47}$ are testing the combination of PARPi with ARtargeted therapy to increase the cancer cells sensivity to PARPi, and another group is testing PARPi plus radiation due to their synergistic impact on cell death. ${ }^{48,49}$

PARPi leading to absence of DNA repair and cell death, will increase the antigenicity as there will be increased DSB and this enhanced antigenicity may increase the efficacy of immune check point inhibitors. Therefore, studies are going on regarding combination therapy of olaparib with durvalumab (PD-L1 inhibitor) in prostate cancer (NCT02484404). At 2017 Annual meeting of American Society of Clinical Oncology, preliminary results were presented, which showed $>50$ reduction in PSA in $43 \%$ patients who got treatment for 2 months. ${ }^{50}$

\section{PARP inhibition in GI malignancies}

Gastric cancer: There is a low prevalence of BRCA mutations in gastric cancer. Low ataxia telangiectasia mutated (ATM) levels have been shown to be associated with olaparib sensitivity in gastric cancer cell lines. ${ }^{51}$ Thirteen percent to $22 \%$ of tumors from patients with gastric cancer have low or undetectable ATM expression, ${ }^{52}$ so may be an attractive target for PARP inhibition.

A phase II trial evaluated the efficacy of olaparib combined with paclitaxel as second-line therapy in Asian patients with advanced gastric cancer. ${ }^{53}$ One hundred and twenty-four patients were randomly assigned to paclitaxel with olaparib vs. paclitaxel with placebo. Fourteen-percent of patients had ATM low gastric tumors. Olaparib did not lead to a significant improvement in PFS; however, it significantly improved OS vs. placebo in both the overall population (13.1 vs. 8.3 months, $\mathrm{P}=0.005$ ) and the ATM low population (not reached vs. 8.2 months, $\mathrm{P}=0.002$ ). On the other hand, in the phase III GOLD trial, olaparib/paclitaxel did not meet its primary endpoint of improvement in OS..$^{53}$ The study randomized 525 Asian patients with advanced gastric cancer to olaparib plus weekly paclitaxel or paclitaxel alone. Median OS was 8.8 months in the olaparib arm and 6.9 months in the placebo arm $(\mathrm{HR}=0.79, \mathrm{P}=0.0262)$. In ATM proteinnegative patients $(\mathrm{n}=94)$, OS was 12.0 vs. 10.0 months, respectively ( $\mathrm{HR}=0.73, \mathrm{P}=0.2458)$. The lack of benefit was seen in both the entire study population and in patients selected for ATM protein negativity.

Pancreatic cancer: Familial pancreas cancer is associated with BRCA1 and BRCA2 mutations [54]. Among patients with FPC, $3.7 \%-5.6 \%$ carry BRCA2 mutations, and $1.2 \%$ carry BRCA1 mutations [55]. Hu et al [56] reported even higher prevalence (14\%) of mutations in 4 selected genes (BRCA1, BRCA2, ATM, and MSH6). The optimal chemotherapy regimen is not known for these patients and currently they are treated with the same regimens we use for patients with no mutations. There are reports showing that they may be more susceptible to platinum-containing chemotherapy regimens.

Several clinical trials of PARP inhibitors are underway testing PARPi maintenance therapy after platinum-based induction regimen. The results of the RUBRACA trial were recently reported in AACR 2019 meeting which showed PFS of 9.1 months, and median overall survival has not been reached yet. RUCAPANC trial reported 32\% ORR among patients with APC harboring BRCA1/BRCA2 mutation. ${ }^{57}$ Veliparib on the other hand has not shown any benefit among this patient population..$^{58,59}$

Phase III POLO trial ${ }^{60}$ APC patients with germline BRCA1/ BRCA2 mutated APC were treated with olaparib $300 \mathrm{mg}$ twice daily or placebo after $\geq 16$ weeks of platinum-based regimen. PFS were improved in olaparib arm versus placebo arm (7.4 months versus 3.8 months, $\mathrm{HR}=0.53, \mathrm{p}=0.004)$. On the other hand, OS was not different (18.9 months versus 18.1 months, $\mathrm{HR}=0.91, \mathrm{p}=0.68$ ).

Veliparib plus GEM/CDDP was studied in patients with untreated germline BRCA1/BRCA2 mutated APC. The ORR was $78 \%$ with median OS of 23 months in BRCA-mutated cases. ${ }^{61} \mathrm{~A}$ randomized phase II trial of GEM/CDDP with or without veliparib is being performed for APC patients both harboring BRCA mutation as well as PALB2 mutation (NCT01585805).

\section{PARP inhibition in small cell lung cancer (SCLC)}

PARP protein levels are upregulated in SCLC relative to other lung cancers. ${ }^{62}$ In particular, PARP1 has been found to be highly expressed at both the mRNA and protein levels in SCLC samples. In a phase I study of extensive stage SCLC, patients were treated with talazoparib monotherapy; ORR was $9 \%$ and the clinical benefit rate at $\geq 16$ weeks was $26 \% .{ }^{63}$ Clinical trials of a number of PARPi, in a range of different treatment settings, are ongoing in patients with SCLC. 
BRCA1/2 mutations are notably rare in primary human SCLCs, occurring in $<2 \%$ of cases, ${ }^{64}$ and HRD-assay scores do not seem to correlate with sensitivity to PARPi in SCLC cell lines. ${ }^{65}$ A distinct mechanism, high expression levels of schlafen family member 11 (SLFN11), has been identified as a critical determinant of PARPi sensitivity in SCLC cell lines and patient-derived xenografts. In a randomized phase II clinical trial, SCLC patients treated with temozolomide and veliparib who had favorable ORR, PFS, and OS had high levels of SLFN11 expression. ${ }^{66}$ The utility of SLFN11 expression as a predictive biomarker for PARPi therapy in SCLC will require validation in prospective trials.

\section{Toxicities}

Each of the three different PARP inhibitors has its own specific indications and individual toxicity profiles. ${ }^{67}$ With olaparib, the most commonly reported AE include nausea (59-78\%), fatigue (41-65\%), vomiting (34-50\%), and anemia (12-32\%). ${ }^{29,32}$ The olaparib-related MDS/AML is rare, occurring in $1 \%$ of patients treated with this agent which can happen from 6 months to 2 years' after therapy. ${ }^{68}$ It is only seen in gBRCA-mutation carriers.

The dose-limiting toxicity (DLT) profile of veliparib is different from that of olaparib which include more nausea/vomiting and seizures. ${ }^{69}$ The reported AEs for veliparib in phase II trials are generally low grade and include fatigue (6\%), nausea (4\%), and leukopenia $(2 \%) .{ }^{35}$ The most common side effects of niraparib are fatigue, pneumonitis, and thrombocytopenia in the phase I trial. ${ }^{36,70}$ Talazoparib has a toxicity profile very similar to that of chemotherapy. ${ }^{63,71}$

CYP3A inhibitors and/or inducers should not be used with olaparib, as olaparib is primarily metabolized by the CYP3A enzymes. ${ }^{72}$ Furthermore, since thrombocytopenia is common with niraparib and talazoparib, antiplatelet and anticoagulation drugs should be avoided. ${ }^{36}$

\section{Mechanisms of resistance}

$15 \%$ of ovarian cancer patients with BRCA-deficient tumors remain disease free for more than 5years from the beginning of the olaparib therapy, ${ }^{32}$ so many patients develop resistance. Several mechanisms have been postulated including new shifting mutations in BRCA1/2 genes; ${ }^{73}$ using ATM-dependent HR repair pathway; ${ }^{74}$ and increased expression of BRCA1, RAD54L, and RMI2 proteins. $^{75}$

\section{Conclusion}

Compelling clinical data, particularly in ovarian cancer, led to reevaluation of these agents in various cancers. The approval of these agents in metastatic gBRCA mutated breast cancer and in first line (germline or somatic BRCA mutated), second line maintenance (with or without BRCA mutation) and palliative settings in gBRCA mutated ovarian cancer and promising results in other solid cancers has created enormous enthusiasm in practicing clinicians to incorporate this drug in their therapeutic armamentarium. Although to date, there is no data demonstrating an OS benefit for PARP inhibitors in breast cancer patients, none of the studies having been powered to detect OS. The ongoing phase III trial OlympiA is powered to assess OS in patients with HER2-negative breast cancer with gBRCA1/2 mutations treated with olaparib in the adjuvant setting; data are expected in 2020. Further accumulation of evidence may yield OS benefit through the studies incorporating OS as the primary end point.

\section{Future perspectives}

Future studies are required to better define which patients to treat with PARPi and whether the target population can be extended beyond those with gBRCA mutations. ${ }^{36}$ It will also be important to assess when to treat these patients; how to sequence these drugs with currently established therapies, particularly platinum agents; how to combine PARPi with other novel therapies; and how to overcome resistance. The data so far suggests that platinum sensitivity predicts response to PARP inhibitors, PARP inhibitors may be useful radiosensitizers and chemosensitizers, and that intermittent dosing and G-CSF support are feasible tactics to mitigate myelosuppressive toxicities of PARP inhibitors when combined with cytotoxic chemotherapies.

\section{Acknowledgments}

None.

\section{Conflicts of interest}

The authors declare there is no conflict of interest.

\section{References}

1. Chambon P, Weill JD, Mandel P. Nicotinamide mononucleotide activation of new DNA-dependent polyadenylic acid synthesizing nuclear enzyme. Biochem Biophys Res Commun. 1963;11:39-43.

2. Vyas $\mathrm{S}$, Chesarone-Cataldo M, Todorova $\mathrm{T}$, et al. A systematic analysis of the PARP protein family identifies new functions critical for cell physiology. Nat Commun. 2013;4:2240.

3. Bayraktar S, Glück S. Systemic therapy options in BRCA mutationassociated breast cancer. Breast Cancer Res Treat. 2012;135(2):355366.

4. Bayraktar S, Glück S. Molecularly targeted therapies for metastatic triple-negative breast cancer. Breast Cancer Res Treat. 2013;138(1):2135.

5. Satoh MS, Lindahl T. Role of poly (ADP-ribose) formation in DNA repair. Nature. 1992;356(6367):356-358.

6. Caldecott KW. Single-strand break repair and genetic disease. Nat Rev Genet. 2008;9(8):619-631.

7. Mao Z, Bozzella M, Seluanov A, et al. Comparison of nonhomologous end joining and homologous recombination in human cells. DNA Repair (Amst). 2008;7(10):1765-1771.

8. Prakash R, Zhang Y, Feng W, et al. Homologous recombination and human health: the roles of BRCA1, BRCA2, and associated proteins. Cold Spring Harb Perspect Biol. 2015;7(4):a016600.

9. Farmer $\mathrm{H}, \mathrm{McCabe} \mathrm{N}$, Lord $\mathrm{CJ}$, et al. Targeting the DNA repair defect in BRCA mutant cells as a therapeutic strategy. Nature. 2005;434(7035):917-921.

10. Turner NC, Lord CJ, Iorns E, et al. A synthetic lethal siRNA screen identifying genes mediating sensitivity to a PARP inhibitor. EMBO J. 2008;27(9):1368-1377.

11. Basourakos SP, Li L, Aparicio AM, et al. Combination platinum-based and DNA damage response-targeting cancer therapy: evolution and future directions. Curr Med Chem. 2017;24(15):1586-1606.

12. Murai J, Huang SY, Renaud A, et al. Stereospecific PARP trapping by BMN 673 and comparison with olaparib and rucaparib. Mol Cancer Ther. 2014;13(2):433-443. 
13. Arun B, Akar U, Gutierrez-Barrera AM, et al. The PARP inhibitor AZD2281 (Olaparib) induces autophagy/mitophagy in BRCA1 and BRCA2 mutant breast cancer cells. Int J Oncol. 2015;7(1):262-268.

14. Pommier Y, O’Connor MJ, de Bono J. Laying a trap to kill cancer cells: PARP inhibitors and their mechanisms of action. Sci Transl Med. 2016;8(362):362ps17.

15. Shen Y, Aoyagi-Scharber M. Wang B. Trapping poly(ADP-Ribose) polymerase. J Pharmacol Exp Ther. 2015;353(3):446-457.

16. Wahlberg E, Karlberg T, Kouznetsova E, et al. Family-wide chemical profiling and structural analysis of PARP and tankyrase inhibitors. Nat Biotechnol. 2012;30(3):283-238.

17. Tutt A, Robson M, Garber JE, et al. Oral poly (ADP-ribose) polymerase inhibitor olaparib in patients with BRCA1 or BRCA2 mutations and advanced breast cancer: a proof-of-concept trial. Lancet. 2010;376(9737):235-244.

18. Gelmon KA, Tischkowitz M, Mackay H, et al. Olaparib in patients with recurrent high-grade serous or poorly differentiated ovarian carcinoma or triple-negative breast cancer: a phase 2, multicentre, open-label, nonrandomised study. Lancet Oncol. 2011;12(9):852-861.

19. Robson M, Im SA, Senkus E, et al. Olaparib for metastatic breast cancer in patients with a germline BRCA mutation. $N$ Engl J Med. 2017;377(6):523-533.

20. Litton JK, Rugo HS, Ettl J, et al. Talazoparib in patients with advanced breast cancer and a germline BRCA mutation. $N$ Engl J Med. 2018;379(8):753-763.

21. A pilot study of neoadjuvant talazoparib for early-stage breast cancer patients with a BRCA mutation, in ESMO 2016 Congress. 2016:43-67.

22. Loibl S, O'Shaughnessy J, Untch M, et al. Addition of the PARP inhibitor veliparib plus carboplatin or carboplatin alone to standard neoadjuvant chemotherapy in triple-negative breast cancer (BrighTNess): a randomised, phase 3 trial. Lancet Oncol. 2018;19(4):497-509.

23. Phase II BROCADE trial. 2017. p. Abstract S 2-5.

24. Domchek SM. Reversion mutations with clinical use of PARP inhibitors: many genes, many versions. Cancer Discov. 2017;7(9):937-939.

25. Moschetta M, George A, Kaye SB, et al. BRCA somatic mutations and epigenetic BRCA modifications in serous ovarian cancer. Ann Oncol. 2016;27(8):1449-1455.

26. Pennington KP, Walsh T, Harrell MI, et al. Germline and somatic mutations in homologous recombination genes predict platinum response and survival in ovarian, fallopian tube, and peritoneal carcinomas. Clin Cancer Res. 2014;20(3):764-775.

27. Elvin JA, Gay LM, Ort R, et al. Clinical benefit in response to palbociclib treatment in refractory uterine leiomyosarcomas with a common. Oncologist. 2017;22(4):416-421.

28. Norquist BM, Harrell MI, Brady MF, et al., Inherited mutations in women with ovarian carcinoma. JAMA Oncol. 2016;2(4):482-490.

29. Kaufman B, , Shapira-Frommer R, Schmutzler RK, et al. Olaparib monotherapy in patients with advanced cancer and a germline BRCA1/2 mutation. J Clin Oncol. 2015;33(3):244-250.

30. Moore K, Colombo N, Scambia G, et al. Maintenance Olaparib in Patients with Newly Diagnosed Advanced Ovarian Cancer. $N$ Engl J Med. 2018;379(26):2495-2505.

31. Pujade-Lauraine E, Ledermann JA, Selle F, et al. Olaparib tablets as maintenance therapy in patients with platinum-sensitive, relapsed ovarian cancer and a BRCA1/2 mutation (SOLO2/ENGOT-Ov21): a double-blind, randomised, placebo-controlled, phase 3 trial. Lancet Oncol. 2017;18(9):1274-1284.

32. Ledermann JA, Harter P, Gourley C, et al. Overall survival in patients with platinum-sensitive recurrent serous ovarian cancer receiving olaparib maintenance monotherapy: an updated analysis from a randomised, placebo-controlled, double-blind, phase 2 trial. Lancet Oncol. 2016;17(11):1579-1589.

33. Richard $\mathrm{T}$ Penson, Ricardo Villalobos Valencia, David Cibula, et al. Olaparib monotherapy versus (vs) chemotherapy for germline BRCA-mutated (gBRCAm) platinum-sensitive relapsed ovarian cancer (PSR OC) patients (pts): Phase III SOLO3 trial. J Clin Oncol. 2019;37(15):5506.

34. Swisher EM, Lin KK, Oza AM, et al. Rucaparib in relapsed, platinum-sensitive high-grade ovarian carcinoma (ARIEL2 Part 1): an international, multicentre, open-label, phase 2 trial. Lancet Oncol. 2017;18(1):75-87.

35. Coleman RL, Oza AM, Lorusso D, et al., Rucaparib maintenance treatment for recurrent ovarian carcinoma after response to platinum therapy (ARIEL3): a randomised, double-blind, placebo-controlled, phase 3 trial. Lancet. 2017;390(10106):1949-1961.

36. Mirza MR, Monk BJ, Herrstedt J, et al. Niraparib maintenance therapy in platinum-sensitive, recurrent ovarian cancer. $N$ Engl J Med. 2016;375(22):2154-2164.

37. Fabbro M, Moore KN, Dørum A, et al. Efficacy and safety of niraparib as maintenance treatment in older patients $(\geq 70$ years) with recurrent ovarian cancer: Results from the ENGOT-OV16/NOVA trial. Gynecol Oncol. 2019;152(3):560-567.

38. Mersch J, Jackson MA, Park M, et al. Cancers associated with BRCA1 and BRCA2 mutations other than breast and ovarian. Cancer. 2015;121(2):269-275.

39. Castro E, Goh C, Olmos D, et al. Germline BRCA mutations are associated with higher risk of nodal involvement, distant metastasis, and poor survival outcomes in prostate cancer. $J$ Clin Oncol. 2013;31(14):1748-1757.

40. Pritchard CC, Mateo J, Walsh MF, et al. Inherited DNA-Repair gene mutations in men with metastatic prostate cancer. $N$ Engl $\mathrm{J}$ Med. 2016;375(5):443-453.

41. Isaacsson Velho P, Silberstein JL, Markowski MC, et al. Intraductal/ ductal histology and lymphovascular invasion are associated with germline DNA-repair gene mutations in prostate cancer. Prostate. 2018;78(5):401-407.

42. Mendes-Pereira AM, Martin SA, Brough R, et al. Synthetic lethal targeting of PTEN mutant cells with PARP inhibitors. EMBO Mol Med. 2009;1(6-7):315-322.

43. Mateo J, Carreira S, Sandhu S, et al. DNA-repair defects and olaparib in metastatic prostate cancer. $N$ Engl J Med. 2015;373(18):1697-708.

44. Mateo J, Porta N, McGovern UB, et al. TOPARP-B: A phase II randomized trial of the PARP inhibitor olaparib for $\mathrm{mCRPC}$ with DNA damage repair alterations. Journal of Clinical Oncology: ASCO Annual Meeting. 2019.

45. Abida W, Bryce AH, Vogelzang NJ, et al. Preliminary results from TRITON2: a phase 2 study of rucaparib in patients (pts) with metastatic castration-resistant prostate cancer (mCRPC). Annals of Oncology: ESMO 2018 Congress. 2018.

46. Schiewer MJ, Goodwin JF, Han S, et al. Dual roles of PARP-1 promote cancer growth and progression. Cancer Discov. 2012;2(12):1134-1149.

47. Asim M, Tarish F, Zecchini HI, et al. Synthetic lethality between androgen receptor signalling and the PARP pathway in prostate cancer. Nat Commun. 2017;8(1):374.

48. Rae C, Mairs RJ. Evaluation of the radiosensitizing potency of chemotherapeutic agents in prostate cancer cells. Int $J$ Radiat Biol. 2017;93(2):194-203. 
49. Steinberger AE, Cotogno P, Ledet EM, et al. Exceptional duration of radium-223 in prostate cancer with a BRCA2 mutation. Clin Genitourin Cancer. 2017;15(1):e69-e71.

50. Karzai F, Ravi AM, Owens H, et al. A phase II study of the antiprogrammed death ligand-1 antibody durvalumab (D; MEDI4736) in combination with PARP inhibitor, olaparib $(\mathrm{O})$, in metastatic castrationresistant prostate cancer (mCRPC). Journal of Clinical Oncology: ASCO Annual Meeting. 2017. 162 p.

51. Hodgson D, H Mason, L Oplustilova, et al. Activity of the PARP inhibitor olaparib in ATM-deficient gastric cancer: from preclinical models to the clinic. Proc Am Assoc Cancer Res: AACR Annual Meeting. 2014

52. Kim HS, Kim MA, Hodgson D, et al. Concordance of ATM (ataxia telangiectasia mutated) immunohistochemistry between biopsy or metastatic tumor samples and primary tumors in gastric cancer patients. Pathobiology. 2013;80(3):127-137.

53. Bang Y, Boku N, Chin K, et al. Olaparib in combination with paclitaxel in patients with advanced gastric cancer who have progressed following first-line therapy: Phase III GOLD trial. Annals of Oncology. 2016:1-36.

54. Leung K, Saif MW. BRCA-associated pancreatic cancer: the evolving management. JOP. 2013;14(2):149-151.

55. Takai E, Yachida S, Shimizu K, et al. Germline mutations in Japanese familial pancreatic cancer patients. Oncotarget. 2016;7(45):7422774235 .

56. Hu C, Hart SN, Bamlet WR, et al. Prevalence of Pathogenic Mutations in Cancer Predisposition Genes among Pancreatic Cancer Patients. Cancer Epidemiol Biomarkers Prev. 2016;25(1):207-211.

57. Shroff RT, Hendifar A, McWilliams RR, et al. Rucaparib monotherapy in patients with pancreatic cancer and a known deleterious BRCA mutation. JCO Precis Oncol. 2018.

58. Lowery MA, Kelsen DP, Capanu M, et al. Phase II trial of veliparib in patients with previously treated BRCA-mutated pancreas ductal adenocarcinoma. Eur J Cancer. 2018;89:19-26.

59. Ohmoto A, Yachida S. Current status of poly(ADP-ribose) polymerase inhibitors and future directions. Onco Targets Ther. 2017;10:5195-5208.

60. Kindler HL HP, Reni M. Olaparib as maintenance treatment following first-line platinum-based chemotherapy $(\mathrm{PBC})$ in patients (pts) with a germline BRCA mutation and metastatic pancreatic cancer ( $\mathrm{mPC}$ ): Phase III POLO trial. J Clin Oncol: ASCO Annual Meeting. 2019.

61. Reilly O, Lee JW, Lowery MA, et al., Phase 1 trial evaluating cisplatin, gemcitabine, and veliparib in 2 patient cohorts: Germline BRCA mutation carriers and wild-type BRCA pancreatic ductal adenocarcinoma. Cancer. 2018;124(7):1374-1382.

62. Byers LA, Wang J, Nilsson MB, et al. Proteomic profiling identifies dysregulated pathways in small cell lung cancer and novel therapeutic targets including PARP1. Cancer discov. 2012;2(9):798-811.

63. de Bono J, Ramanathan RK, Mina L, et al. Phase I, Dose-Escalation, Two-Part Trial of the PARP Inhibitor Talazoparib in Patients with Advanced Germline. Cancer Discov. 2017;7(6):620-629.

64. George J, Lim JS, Jang SJ, et al. Comprehensive genomic profiles of small cell lung cancer. Nature. 2015;524(7563):47-53.

65. Lok BH, Gardner EE, Schneeberger VE, et al. PARP inhibitor activity correlates with SLFN11 expression and demonstrates synergy with temozolomide in small cell lung cancer. Clin Cancer Res. 2017;23(2):523-535.

66. Byers LA. Improved small cell lung cancer response rates with veliparib and temozolomide: results from a phase II trial. J Thorac Oncol. 2016;12:S406-S407.

67. La Fargue CJ, Dal Molin GZ, Sood AK, et al. Exploring and comparing adverse events between PARP inhibitors. Lancet Oncol. 2019;20(1):e15e28

68. Ricks TK, Chiu HJ, Ison G, et al. Successes and Challenges of PARP Inhibitors in Cancer Therapy. Front Oncol. 2015;5:222.

69. Puhalla SBJ, Pahuja S, Appleman LJ, et al. Final results of a phase 1 study of single-agent veliparib (V) in patients (pts) with either BRCA1/2-mutated cancer (BRCA?), platinum-refractory ovarian, or basal-like breast cancer (BRCA-wt). ASCO Annual Proceedings. 2014.

70. Sandhu SK, Schelman WR, Wilding G, et al. The poly(ADP-ribose) polymerase inhibitor niraparib (MK4827) in BRCA mutation carriers and patients with sporadic cancer: a phase 1 dose-escalation trial. Lancet Oncol. 2013;14(9):882-892.

71. Zhou JX, Feng LJ, Zhang X, et al. Risk of severe hematologic toxicities in cancer patients treated with PARP inhibitors: a meta-analysis of randomized controlled trials. Drug Des Devel Ther. 2017;11:3009-3017.

72. Dirix L, Swaisland H, Verheu HM, et al. Effect of Itraconazole and Rifampin on the Pharmacokinetics of Olaparib in Patients With Advanced Solid Tumors: Results of Two Phase I Open-label Studies. Clin Ther. 2016;38(10):2286-2299.

73. Sakai W, Swisher EM, Karlan BY, et al. Secondary mutations as a mechanism of cisplatin resistance in BRCA2-mutated cancers. Nature. 2008; 451(7182):1116-1120.

74. Montoni A, Robu M, Pouliot E, et al. Resistance to PARP-inhibitors in cancer therapy. Front Pharmacol. 2013;4:18.

75. Li L, Karanika S, Yang G, et al. Androgen receptor inhibitor-induced "BRCAness" and PARP inhibition are synthetically lethal for castrationresistant prostate cancer. Sci Signal. 2017;10(480). 